\title{
The cosmological constant in supergravity
}

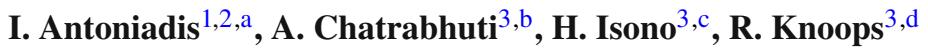 \\ ${ }^{1}$ Laboratoire de Physique Théorique et Hautes Énergies, LPTHE, Sorbonne Université, CNRS, 4 Place Jussieu, 75005 Paris, France \\ ${ }^{2}$ Albert Einstein Center, Institute for Theoretical Physics, University of Bern, Sidlerstrasse 5, 3012 Bern, Switzerland \\ ${ }^{3}$ Department of Physics, Faculty of Science, Chulalongkorn University, Phayathai Road, Pathumwan, Bangkok 10330, Thailand
}

Received: 16 May 2018 / Accepted: 22 August 2018 / Published online: 5 September 2018

(C) The Author(s) 2018

\begin{abstract}
We propose a supersymmetrisation of the cosmological constant in ordinary $N=1$ supergravity that breaks supersymmetry spontaneously by a constant FayetIliopoulos (FI) term associated to a $U(1)$ symmetry. This term is a variation of a new gauge invariant FI term proposed recently, which is invariant under Kähler transformations and can be written even for a gauged R-symmetry on top of the standard FI contribution. The two terms are the same in the absence of matter but differ in its presence. The proposed term is reduced to a constant FI-term up to fermion interactions that disappear in the unitary gauge in the absence of any F-term supersymmetry breaking. The constant FI term leads to a positive cosmological constant, uplifting the vacuum energy from the usual anti-de Sitter supergravity to any higher value.
\end{abstract}

\section{Introduction}

It is well known that the cosmological constant $\Lambda$ in supergravity is highly constrained. For given gravitino mass term $m_{3 / 2}$, there is a lowest value of $\Lambda=-3 m_{3 / 2}^{2}$ corresponding to the anti de Sitter (AdS) supergravity, describing a massless spin-3/2 spinor in AdS [1]. It is obtained in the absence of matter fields by a constant superpotential. Uplifting this value breaks supersymmetry and can be done in principle dynamically by minimising a scalar potential. Supersymmetry breaking then occurs by a vacuum expectation value (VEV) of an F-auxiliary component of a chiral superfield containing the goldstino.

\footnotetext{
a e-mail: antoniadis@itp.unibe.ch

be-mail:dma3ac2@gmail.com

c e-mail: hiroshi.isono81@gmail.com

de-mail: rob.k@chula.ac.th
}

In the absence of matter, one could still break supersymmetry by a VEV of a D-auxiliary component of a vector superfield which requires the addition of a constant FayetIliopoulos (FI) contribution [2]. This can be done only when the vector superfield gauges the R-symmetry, under which the chiral compensator of $N=1$ supergravity becomes charged [3,4]. A constant superpotential is however forbidden in that case, since it must be charged under R-symmetry, and there is no explicit gravitino mass term, although supersymmetry is broken in de Sitter (dS) space. In the presence of matter, a charged superpotenial can be written but then supesymmetry is also broken by the VEV of a chiral multiplet upon minimisation of the corresponding scalar potential. Thus, the cosmological constant cannot be added as an independent parameter in supergravity for arbitrary breaking scale (gravitino mass).

An exception to the above situation is when supersymmetry is non-linearly realized by introducing a constrained goldstino superfield $X$ satisfying the nilpotent condition $X^{2}=0$ [5-7]. This eliminates the scalar component (sgoldstino) in terms of the goldstino bilinear and the scalar potential (in the absence of matter) becomes an arbitrary constant uplifting the minimal value $\Lambda=-3 m_{3 / 2}^{2}$ [7]. However in this case supersymmetry is not spontaneously broken in a linear way and the number of bosonic and fermionic degrees of freedom are not equal, invalidating in particular the usual ultraviolet properties of $N=1$ supergravity and of its low energy softly broken supersymmetric theory.

Recently, a new FI term was proposed that allows an arbitrary uplifting of the vacuum energy in the absence of matter fields $[8,9] .{ }^{1}$ It does not require gauging the R-symmetry and in the unitary (super)gauge of massive gravitino, it is reduced to just an additive positive constant to $\Lambda$. In the presence

\footnotetext{
1 Another approach to the new FI term was proposed in [10].
} 
of matter, however, it leads to an additional field-dependent contribution to the scalar potential. Moreover, it breaks the invariance of the standard two-derivative supergravity action under Kähler transformations.

In this work, we propose a modification/generalisation of this FI term that has the following properties: (1) it can be written independently whether the corresponding $U(1)$ gauges or not the R-symmetry; (2) in the absence of matter fields and in the case of an ordinary (non-R) $U(1)$, it coincides with the one proposed in [8]; (3) in the case of a $U(1)_{R}$, it can be written on top of the standard constant FI term; (4) in the presence of matter fields the action is invariant under Kähler transformations and its bosonic contribution is always a constant FI term that uplifts in particular the vacuum energy by an arbitrary positive constant.

The outline of the paper is the following. In Sect. 2, we review the new FI term and its properties. In Sect. 3, we present a modification/generalisation that is invariant under Kähler transformations. In Sect. 4, we compute its bosonic contribution to the standard supergravity action. Section 5 contains some concluding remarks. Finally, we have two appendices; "Appendix A" contains useful formulae used in the text, while in "Appendix B" we compute the fermionic part of the supergravity action.

\section{Review and definitions}

In [8] a new FI term has been proposed of the form

$$
\mathcal{L}_{\mathrm{FI}}=-\xi\left[S_{0} \bar{S}_{0} \frac{w^{2} \bar{w}^{2}}{T\left(\bar{w}^{2}\right) \bar{T}\left(w^{2}\right)}(V)_{D}\right]_{D},
$$

where we put the Planck mass to 1 , and $\xi$ is a constant parameter. We use the conventions of $[1,11]$. Here $S_{0}=$ $\left(s_{0}, P_{L} \Omega_{0}, F_{0}\right)$ and $\bar{S}_{0}=\left(\bar{s}_{0}, P_{R} \Omega_{0}, \bar{F}_{0}\right)$ are the chiral (and anti-chiral) compensator fields with (Weyl, Chiral) weights $(1,1)$ and $(1,-1)$ respectively, and $V$ is a real (vector) supermultiplet with weights $(0,0)$ and components $V=$ $\left(v, \zeta, \mathcal{H}, A_{\mu}, \lambda, D\right)$, where the first three components are zero in the Wess-Zumino gauge $v=\zeta=\mathcal{H}=0$. The linear projection $(V)_{D}$ has weights $(2,0)$ and is defined by

$$
(V)_{D}=\left(D, \not D \lambda, 0, \mathcal{D}^{b} \hat{F}_{a b},-\not D D D \lambda,-\square^{C} D\right)
$$

The chiral (and anti-chiral) multiplets $w^{2}$ (and $\bar{w}^{2}$ ) are given by

$$
w^{2}=\frac{\bar{\lambda} P_{L} \lambda}{S_{0}^{2}}, \quad \bar{w}^{2}=\frac{\lambda P_{R} \bar{\lambda}}{\bar{S}_{0}^{2}},
$$

where the components of $\bar{\lambda} P_{L} \lambda$ are, ${ }^{2}$

$$
\begin{aligned}
\bar{\lambda} P_{L} \lambda= & \left(\bar{\lambda} P_{L} \lambda ; \sqrt{2} P_{L}\left(-\frac{1}{2} \gamma \cdot \hat{F}+i D\right) \lambda ;\right. \\
& \left.2 \bar{\lambda} P_{L} \not D \lambda+\hat{F}^{-} \cdot \hat{F}^{-}-D^{2}\right)
\end{aligned}
$$

where the self-dual and anti-self-dual tensors are defined by

$\hat{F}_{\mu \nu}^{ \pm}=\frac{1}{2}\left(\hat{F}_{\mu \nu} \pm \tilde{\hat{F}}_{\mu \nu}\right), \quad \tilde{\hat{F}}_{\mu \nu}=-\frac{1}{2} i \epsilon_{\mu \nu \rho \sigma} \hat{F}^{\rho \sigma}$,

and the definitions of the covariant field strength $\hat{F}$ and the covariant derivative $\not{D} \lambda$ can be found in "Appendix A". The conformal d'Alembertian is given by $\square^{C}=\eta^{a b} \mathcal{D}_{a} \mathcal{D}_{b}$. Note that $w^{2}$ has weights $(1,1), \bar{w}^{2}$ has weights $(1,-1)$, and $\bar{\lambda} P_{L} \lambda$ has weights $(3,3)$.

The chiral (and anti-chiral) projection operators $T$ (and $\bar{T}$ ) are defined in $[11,12]$. In particular if $\mathcal{C}$ is a general (unconstrained) multiplet of weights $(\omega, \omega-2)$ given by

$\mathcal{C}=\left(\mathcal{C}, \mathcal{Z}, \mathcal{H}, \mathcal{K}, \mathcal{B}_{a}, \Lambda, \mathcal{D}\right)$

then $T(\mathcal{C})$ has weights $(\omega+1, \omega+1)$ and is given by

$T(\mathcal{C})=\left(-\frac{1}{2} \mathcal{K},-\frac{1}{2} \sqrt{2} i P_{L}(\not D \mathcal{Z}+\Lambda), \frac{1}{2}\left(\mathcal{D}+\square^{C} \mathcal{C}+i \mathcal{D}_{a} \mathcal{B}^{a}\right)\right)$.

The resulting chiral multiplet $T(C)$ has weights $(\omega+1, \omega+$ 1). The operation $T$ acting on a chiral multiplet $X=$ $\left(\phi, P_{L} \chi, F\right)$ vanishes, i.e. $T(X)=0$, while its action on an anti-chiral multiplet $\bar{X}=\left(\bar{\phi}, P_{R} \chi, \bar{F}\right)$ of weights $(1,-1)$ is defined as

$T(\bar{X})=\left(\bar{F}, \not D P P_{R} \chi, \square^{C} \bar{\phi}\right)$

For more information, the reader is referred to "Appendix A.1". In rigid supersymmetry, this corresponds to the usual chiral (and anti-chiral) projection operators $\bar{D}^{2}$ (and $D^{2}$ ).

For simplicity, we assume a constant gauge kinetic function. The kinetic terms for the gauge multiplet are given by

$\mathcal{L}_{\text {kin }}=-\frac{1}{4}\left[\bar{\lambda} P_{L} \lambda\right]_{F}$

The extension to a non-trivial gauge kinetic function is given in [9].

The operation []$_{F}$ acts on a chiral multiplet $X=$ $\left(\phi, P_{L} \Omega, F\right)$ with weights $(3,3)$, giving $[11],{ }^{3}$

\footnotetext{
${ }^{2}$ Note that in this notation the field strength superfield $\mathcal{W}_{\alpha}$ is given by $\mathcal{W}^{2}=\bar{\lambda} P_{L} \lambda$, and $(V)_{D}$ corresponds to $\mathcal{D}^{\alpha} \mathcal{W}_{\alpha}$.

${ }^{3}$ Note that the definitions of []$_{F}$ and []$_{D}$ below do not involve the spacetime integral $\int d^{4} x$, which appears in the corresponding expressions in [11].
} 
$[X]_{F}=e\left[F+\frac{1}{\sqrt{2}} \bar{\psi}_{\mu} \gamma^{\mu} P_{L} \Omega+\frac{1}{2} \phi \bar{\psi}_{\mu} \gamma^{\mu \nu} P_{R} \psi_{\nu}\right]+$ h.c.

Note that this already contains the Hermitian conjugate. The operation [ $]_{D}$ acts on a real multiplet $C=\left(C, \zeta, \mathcal{H}, v_{\mu}, \lambda, D\right)$ of weights $(2,0)$, giving [11]

$$
\begin{aligned}
{[C]_{D}=} & \frac{1}{2} e\left[D-\frac{i}{2} \bar{\psi} \cdot \gamma \gamma_{*} \lambda-\frac{1}{3} C R(\omega)\right. \\
& +\frac{1}{6}\left(C \bar{\psi}_{\mu} \gamma^{\mu \rho \sigma}-i \zeta \gamma^{\rho \sigma} \gamma_{*}\right) R_{\rho \sigma}^{\prime}(Q) \\
& \left.+\frac{1}{4} \epsilon^{a b c d} \bar{\psi}_{a} \gamma_{b} \psi_{c}\left(v_{d}-\frac{1}{2} \bar{\psi}_{d} \zeta\right)\right]
\end{aligned}
$$

Here $\psi$ is the gravitino, and $R(\omega)$ and $R_{\rho \sigma}^{\prime}(Q)$ are the graviton and gravitino curvatures.

The Lagrangian contains a term,

$\mathcal{L}_{\mathrm{FI}} / e=-\xi s_{0} \bar{s}_{0} D$

After the auxiliary field $D$ is integrated out with the contribution from the kinetic term $D^{2} / 2$ taken into account, the scalar potential contains a term proportional to $\xi^{2}$,

$\mathcal{V}_{\mathrm{FI}}=\frac{\xi^{2}}{2}\left(s_{0} \bar{s}_{0}\right)^{2}$

In the absence of additional matter fields, one can use the Poincaré gauge $s_{0}=\bar{s}_{0}=1$, resulting in a constant Dterm contribution to the scalar potential. However, when matter fields are included, the Einstein frame gauge gives $s_{0}=\bar{s}_{0}=e^{K / 6}$ fixing the conformal symmetry, leading to a field dependent FI contribution to the scalar potential:

$\mathcal{V}_{\mathrm{FI}}=\frac{\xi^{2}}{2} e^{2 K / 3}$

The implications of such a term to inflation have been studied in $[9,13]$.

In the presence of matter fields the FI term (1) is not invariant under Kähler transformations. The purpose of this paper is to construct a term, similar to Eq. (1), invariant under Kähler transformations. As a consequence, the contribution to the scalar potential will no longer depend on $e^{2 K / 3}$ as in Eq. (14).

\section{Kähler invariant generalization of the new FI term}

In this section we find a generalization of the new FI term in Eq. (1) that is invariant under Kähler transformations in the presence of matter multiplets. For simplicitly, we denote generically the chiral multiplets by $X$. The standard $N=1$ supergravity Lagrangian is given by
$\mathcal{L}_{X}=-3\left[S_{0} \bar{S}_{0} e^{-K / 3}\right]_{D}+\left[S_{0}^{3} W\right]_{F}$,

for a Kähler potential $K(X, \bar{X})$ and a superpotential $W(X)$. A Kähler transformation with parameter $J(X)$ is given by

$$
\begin{aligned}
K(X, \bar{X}) & \rightarrow K(X, \bar{X})+J(X)+\bar{J}(\bar{X}), \\
W(X) & \rightarrow W(X) e^{-J(X)} \\
S_{0} & \rightarrow S_{0} e^{\frac{J(X)}{3}}
\end{aligned}
$$

It is clear that $\mathcal{L}_{X}$ in Eq. (15) is invariant under Kähler transformations. However, the new FI term proposed in [8] and given in Eq. (1) is not.

In [8] it is suggested that a Kähler invariant generalization can be found by making the FI constant field dependent, i.e. $\xi=\xi(X, \bar{X})$. However, $\bar{T}\left(w^{2}\right)$ is not Kähler covariant since the conformal compensator $S_{0}$ transforms under Kähler transformations. Thus, under a Kähler transformation, $w^{2}$ transforms as $w^{2} \rightarrow w^{2} e^{-2 J / 3}$ while $\bar{T}\left(w^{2} e^{-2 J / 3}\right) \neq$ $\bar{T}\left(w^{2}\right) e^{-2 J / 3}$. Instead, in this paper we keep $\xi$ constant, and modify the new FI term by requiring the compensator fields $S_{0}$ and $\bar{S}_{0}$ to appear in the new FI term only through the Kähler invariant combination $S_{0} \bar{S}_{0} e^{-K / 3}$.

First, recall that the operators $T$ and $\bar{T}$ should act on multiplets with weights $(\omega, \omega-2)$ and $(\omega, 2-\omega)$ respectively. We therefore want to replace $\bar{w}^{2}$ with a multiplet proportional to $\lambda P_{R} \bar{\lambda}$ with weights $(\omega, \omega-2)$. Since $\lambda P_{R} \bar{\lambda}$ is Kähler invariant and has weights $(3,-3)$, we should multiply it with the Kähler invariant combination $S_{0} \bar{S}_{0} e^{-K / 3}$ of weights $(2,0)$ to obtain a multiplet of weights $(\omega, \omega-2)$. Indeed,

$\bar{w}^{\prime 2}=\left(S_{0} \bar{S}_{0} e^{-K / 3}\right)^{m} \lambda P_{R} \bar{\lambda}$

has weights $(2 m+3,-3)$. Thus, $(2 m+3,-3)=(\omega, \omega-2)$ can be solved for $\omega=-1$ and $m=-2$, and one can define a Kähler invariant combination $\bar{w}^{\prime 2}$ with weights $(-1,-3)$,

$\bar{w}^{\prime 2}=\frac{\bar{\lambda} P_{R} \lambda}{\left(S_{0} \bar{S}_{0} e^{-K / 3}\right)^{2}}$.

The resulting $T\left(\bar{w}^{\prime 2}\right)$ has weights $(0,0)$. Similarly, one can construct $\bar{T}\left(w^{\prime 2}\right)$ with weights $(0,0)$, where

$w^{\prime 2}=\frac{\bar{\lambda} P_{L} \lambda}{\left(S_{0} \bar{S}_{0} e^{-K / 3}\right)^{2}}$.

By the same arguments, the new FI contribution to the Lagrangian has the form,

$$
\mathcal{L}_{\mathrm{FI}, \text { new }}=-\xi\left[\left(S_{0} \bar{S}_{0} e^{-K / 3}\right)^{k} \frac{\left(\bar{\lambda} P_{L} \lambda\right)\left(\lambda P_{R} \bar{\lambda}\right)}{T\left(\bar{w}^{\prime 2}\right) \bar{T}\left(w^{\prime 2}\right)}(V)_{D}\right]_{D} .
$$


The operation [ $]_{D}$ is defined only on a multiplet with weights $(2,0)$, from which it follows that $k=-3$.

We conclude that the new FI term is given by,

$\mathcal{L}_{\mathrm{FI}, \text { new }}=-\xi\left[\left(S_{0} \bar{S}_{0} e^{-K / 3}\right)^{-3} \frac{\left(\bar{\lambda} P_{L} \lambda\right)\left(\lambda P_{R} \bar{\lambda}\right)}{T\left(\bar{w}^{\prime 2}\right) \bar{T}\left(w^{\prime 2}\right)}(V)_{D}\right]_{D}$,

with $w^{\prime 2}$ and $\bar{w}^{\prime 2}$ defined in Eqs. (18) and (19) that are invariant under Kähler transformations and have the correct Weyl and Chiral weights. It remains to be shown in the next section that this indeed leads to a constant D-term contribution to scalar potential.

\section{A constant FI contribution to the scalar potential}

In this section we calculate the (purely) bosonic contributions to the D-term scalar potential of the Lagrangian

$\mathcal{L}=\mathcal{L}_{X}+\mathcal{L}_{\text {kin }}+\mathcal{L}_{\mathrm{FI}, \text { new }}$

with the matter contributions $\mathcal{L}_{X}$ given by Eq. (15), the gauge kinetic terms $\mathcal{L}_{\text {kin }}$ given by Eq. (9), and the new FI term $\mathcal{L}_{\text {FI, new }}$ defined in Eq. (21).

For compactness, we put the fermions to zero, and postpone the treatment of the fermion couplings to "Appendix B". The purely bosonic contributions to $\bar{\lambda} P_{L} \lambda$ are

$\bar{\lambda} P_{L} \lambda=\left(0,0, F^{-} \cdot F^{-}-D^{2}\right)$,

while the purely bosonic contributions to $w^{\prime 2}$ are given by, ${ }^{4}$

$$
\begin{aligned}
w^{\prime 2}= & \left(0,0,2\left(s_{0} \bar{s}_{0} e^{-K / 3}\right)^{-2}\left(D^{2}-F^{-} \cdot F^{-}\right), 0\right. \\
& \left.0,0,4\left(s_{0} \bar{s}_{0} e^{-K / 3}\right)^{-2}\left(D^{2}-F^{-} \cdot F^{-}\right)\left(\frac{\bar{F}_{0}}{\bar{s}_{0}}-\frac{K_{\bar{\phi}} \bar{F}}{3}\right)\right) .
\end{aligned}
$$

The bosonic contributions to the anti-chiral projection $\bar{T}\left(w^{\prime 2}\right)$ are

$$
\begin{aligned}
\bar{T}\left(w^{\prime 2}\right)= & \left(\left(s_{0} \bar{s}_{0} e^{-K / 3}\right)^{-2}\left(F^{-} \cdot F^{-}-D^{2}\right), 0,\right. \\
& \left.2\left(s_{0} \bar{s}_{0} e^{-K / 3}\right)^{-2}\left(D^{2}-F^{-} \cdot F^{-}\right)\left(\frac{\bar{F}_{0}}{\bar{s}_{0}}-\frac{K_{\bar{\phi}} \bar{F}}{3}\right)\right) .
\end{aligned}
$$

Here the chiral multiplet $X$ is defined as $X=\left(\phi, P_{L} \chi, F\right)$ and $K_{\phi}=\partial_{\phi} K$.

\footnotetext{
$\overline{{ }^{4} \text { Note that } w^{\prime 2}}$ has seven components since $w^{\prime 2}$ is neither real nor chiral. As for $\bar{w}^{\prime 2}$, its third component vanishes and instead the fourth one becomes the complex conjugate of the third one of $w^{\prime 2}$.
}

Next, we notice that the real multiplet

$\mathcal{R}=\left(S_{0} \bar{S}_{0} e^{-K / 3}\right)^{-3} \frac{\left(\bar{\lambda} P_{L} \lambda\right)\left(\bar{\lambda} P_{R} \lambda\right)}{T\left(\bar{w}^{\prime 2}\right) \bar{T}\left(w^{\prime 2}\right)}$,

is a function of chiral multiplets $Z^{\alpha}=X, S_{0}, \bar{\lambda} P_{L} \lambda, T\left(\bar{w}^{\prime 2}\right)$ and their anti-chiral counterparts $\bar{Z}^{\bar{\alpha}}$. Its components are

$$
\begin{aligned}
\mathcal{R}= & \left(\mathcal{R}, 0,-2 \mathcal{R}_{\alpha} F^{\alpha}, i \mathcal{R}_{\alpha} \mathcal{D}_{\mu} Z^{\alpha}-i \mathcal{R}_{\bar{\alpha}} \mathcal{D}_{\mu} \bar{Z}^{\bar{\alpha}}, 0,\right. \\
& \left.2 \mathcal{R}_{\alpha \bar{\beta}}\left(-\mathcal{D}_{\mu} Z^{\alpha} \mathcal{D}^{\mu} \bar{Z}^{\bar{\beta}}+F^{\alpha} \bar{F}^{\bar{\beta}}\right)\right),
\end{aligned}
$$

where $\mathcal{R}_{\alpha}=\frac{\partial \mathcal{R}}{\partial Z^{\alpha}}$ and all fields are replaced by their lowest components. Note also that obviously fermionic contributions are ignored in Eq. (27). As a result, the components of $\mathcal{R}$ only with bosonic fields are given by,

$\mathcal{R}=\left(0,0,0,0,0,2 s_{0} \bar{s}_{0} e^{-K / 3}\right)$.

It follows that the contribution to the new FI term Lagrangian Eq. (21) is given by,

$\mathcal{L}_{\mathrm{FI}, \text { new }} / e=-\xi s_{0} \bar{s}_{0} e^{-K / 3} D$.

In the Einstein frame gauge $s_{0}=\bar{s}_{0}=e^{K / 6}$, this becomes a constant FI term,

$\mathcal{L}_{\mathrm{FI}, \text { new }} / e=-\xi D$.

We therefore conclude that the Lagrangian of the $\mathrm{U}(1)$ gauge field sector is,

$\left(\mathcal{L}_{\text {kin }}+\mathcal{L}_{\mathrm{FI}, \text { new }}\right) / e=-\frac{1}{4} F_{\mu \nu} F^{\mu v}+\frac{1}{2} D^{2}-\xi D+$ fermions,

which results in a constant FI contribution to the scalar potential. However, the terms in the denominator of Eq. (21) are proportional to $D^{2}-F^{-} \cdot F^{-}$and $D^{2}-F^{+} \cdot F^{+}$(see Eq. (25)). The new FI term is therefore local only if $\langle D\rangle$ is non-vanishing. The theory becomes ill-defined as $\xi \rightarrow 0$, as was the case in [8], since in this limit $\langle D\rangle=0$.

However, in contrast with the term proposed by [8], the term proposed in Eq. (21) is manifestly Kähler and Weyl invariant. While both terms can be easily extended to include charged matter fields, only Eq. (21) is consistent with matter fields that are charged under a gauged R-symmetry, as a consequence of its Kähler invariance.

Therefore, while in [9], the new FI term of [8] could only be added on top of the the usual FI contribution in the Kähler frame where the gauge symmetry is not an R-symmetry, the new FI term in Eq. (21) is consistent with the usual FI contribution in any Kähler frame, resulting in two constant contributions to the D-term contribution in the scalar potential.

A few remarks are in order: 
- Firstly, notice that one could also obtain a constant FI contribution to the D-term by making the substitution $S_{0} \rightarrow S_{0} e^{-K / 6}$ and $\bar{S}_{0} \rightarrow \bar{S}_{0} e^{-K / 6}$ in Eq. (1), giving,

$\mathcal{L}_{\mathrm{FI}, \mathrm{c}}=-\xi\left[S_{0} \bar{S}_{0} e^{-K / 3} \frac{w_{c}^{2} \bar{w}_{c}^{2}}{T\left(\bar{w}_{c}^{2}\right) \bar{T}\left(w_{c}^{2}\right)}(V)_{D}\right]_{D}$,

with,

$w_{c}^{2}=\frac{\bar{\lambda} P_{L} \lambda}{S_{0}^{2} e^{-K / 3}}$

The resulting Lagrangian indeed contains the term $\xi S_{0} \bar{S}_{0} e^{-K / 3} \mathrm{D}$ which results in a constant FI term in the Einstein frame gauge. However, this term is not invariant under Kähler transformations Eq. (16) since $T\left(\bar{w}_{c}^{2}\right)$ does not transform covariantly, and we therefore do not analyse this term further.

- Secondly, note that in the absence of matter fields (and therefore $K(X, \bar{X})=0), w^{2}$ defined in Eq. (3) and $w^{\prime 2}$ defined in Eq. (19) are related by,

$w^{\prime 2}=\bar{S}_{0}^{-2} w^{2}$

By using the property of the anti-chiral projection operators that for an anti-chiral field $\bar{Z}$ and a multiplet $\mathcal{C}$,

$\bar{T}(\bar{Z} \mathcal{C})=\bar{Z} \bar{T}(\mathcal{C})$

we find that,

$\bar{T}\left(w^{\prime 2}\right)=\bar{S}_{0}^{-2} \bar{T}\left(w^{2}\right)$,

and similarly,

$T\left(\bar{w}^{\prime 2}\right)=S_{0}^{-2} T\left(\bar{w}^{2}\right)$.

As a result, in the absence of matter fields, our proposed FI term in Eq. (20) is identical to the one proposed in [8] and given in Eq. (1)..$^{5}$

\section{Conclusions}

In summary, in this work, we proposed a supersymmetrisation of the cosmological constant in $N=1$ supergravity, arising from a constant FI term associated to an abelian gauge symmetry as in global supersymmetry. In contrast to the standard FI term which requires the gauging of R-symmetry, it can be written for any $U(1)$. It is obtained by a variation of a new FI term proposed recently in a way that is invariant

${ }^{5}$ We thank A. Van Proeyen for bringing our attention to this. under Kähler transformations, leading to just a constant FI term up to fermion contributions that disappear in the unitary gauge in the absence of any F-term supersymmetry breaking.

Since a generalisation of such 'new' FI terms is not unique and may in general involve new field dependent functions, ${ }^{6}$ an interesting question is whether they can arise in the effective supergravity of string compactifications and what their form is. An obvious application of the proposed term is that it uplifts the vacuum energy with a positive contribution, allowing to realise 'realistic' models of moduli stabilisation and inflation based on the KKLT mechanism [16] without the need of introducing anti-D3 branes, using a $U$ (1) factor of 'effective' 3-branes gauge group whose gauge coupling is fixed by the ten-dimensional dilaton $[17,18]$.

Acknowledgements We would like to thank Jean-Pierre Derendinger, Sergey Ketov, J. Anibal Sierra-Garcia and Antoine Van Proeyen for fruitful discussions. This work was supported in part by the Swiss National Science Foundation, in part by a CNRS PICS grant and in part by the "CUniverse" research promotion Project by Chulalongkorn University (Grant reference CUAASC).

Open Access This article is distributed under the terms of the Creative Commons Attribution 4.0 International License (http://creativecomm ons.org/licenses/by/4.0/), which permits unrestricted use, distribution, and reproduction in any medium, provided you give appropriate credit to the original author(s) and the source, provide a link to the Creative Commons license, and indicate if changes were made.

Funded by SCOAP ${ }^{3}$.

\section{A Useful formulas}

A.1 The chiral projection and complex multiplets

This Appendix is based on [11]. The operation $T$ acts on a complex multiplet $\mathcal{C}$ with weights (Weyl, Chiral $)=(\omega, \omega-$ $2)$, producing a chiral multiplet with the first component $-\frac{1}{2} \mathcal{K}$. In particular if $\mathcal{C}$ is a general (complex) multiplet given by,

$\mathcal{C}=\left(\mathcal{C}, \mathcal{Z}, \mathcal{H}, \mathcal{K}, \mathcal{B}_{a}, \Lambda, \mathcal{D}\right)$

then $T(\mathcal{C})$ has weights $(\omega+1, \omega+1)$ and is given in Eq. (7), repeated here for convenience, ${ }^{7}$

$T(\mathcal{C})=\left(-\frac{1}{2} \mathcal{K},-\frac{1}{2} \sqrt{2} i P_{L}(\not D \mathcal{Z}+\Lambda), \frac{1}{2}\left(\mathcal{D}+\square^{C} \mathcal{C}+i \mathcal{D}_{a} \mathcal{B}^{a}\right)\right)$

\footnotetext{
${ }^{6}$ It is worth noting that shortly after submitting this paper, several attempts in this direction have been made. See for Example [14] and [15].

7 The three-component notation of chiral/anti-chiral multiplets will be defined shortly.
} 
The anti-chiral projector $\bar{T}(\mathcal{C})$ acts on a multiplet of weights $(\omega, 2-\omega)$ and results in an anti-chiral multiplet of weights $(\omega+1,-\omega-1)$ with lowest component $-\frac{1}{2} \mathcal{H}$.

The restriction of $\mathcal{C}=C$ is real produces a real multiplet. This also implies that the Chiral weight $c=0$. Moreover, $\mathcal{Z}=\eta$ and $\Lambda=\lambda$ are Majorana $\left(P_{R} \mathcal{Z}\right)^{C}=P_{L} \mathcal{Z}$, and $\mathcal{K}=\overline{\mathcal{H}}$, while $\mathcal{B}_{\mu}=B_{\mu}$ and $\mathcal{D}=D$ are real,

$C=\left(C, \zeta, \mathcal{H}, \overline{\mathcal{H}}, B_{\mu}, \lambda, D\right)$

For a real multiplet, we usually abbreviate $\overline{\mathcal{H}}$. The chiral projector $T$ can only act on a real multiplet of weights $(2,0)$.

A chiral multiplet is obtained by the restrictions,

$P_{R} \mathcal{Z}=0, \quad \mathcal{K}=0, \quad \mathcal{B}_{\mu}=i \mathcal{D}_{\mu} \mathcal{C}, \quad \Lambda=0, \quad \mathcal{D}=0$,

and is given by,

$X=\left(X,-i \sqrt{2} P_{L} \chi,-2 F, 0, i \mathcal{D}_{\mu} X, 0,0\right)$.

Similarly an antichiral multiplet is given by,

$\bar{X}=\left(\bar{X}, i \sqrt{2} P_{R} \chi, 0,-2 \bar{F},-i \mathcal{D}_{\mu} \bar{X}, 0,0\right)$.

However, the chiral multiplet in Eq. (42) is usually denoted as $X=\left(X, P_{L} \chi, F\right)$, while the anti-chiral multiplet $\bar{X}=$ $\left(\bar{X}, P_{R} \chi, \bar{F}\right)$. A chiral multiplet has equal chiral and Weyl weights $c=\omega$, and an anti-chiral multiplet has $c=-\omega$. As a result, $T$ can only act on an anti-chiral multiplet of weights $(1,-1)$ and is given by

$T(\bar{X})=\left(\bar{F}, \not D P_{R} \chi, \square^{C} \bar{X}\right)$.

\section{A.2 Covariant derivatives}

The definitions of $\not{D} \lambda$ and the covariant field strength $\hat{F}_{a b}$ can be found in Eq. (17.1) of [1], which reduce for an abelian gauge field to

$$
\begin{aligned}
\hat{F}_{a b}= & e_{a}^{\mu} e_{b}^{\nu}\left(2 \partial_{[\mu} A_{\nu]}+\bar{\psi}_{[\mu} \gamma_{\nu]} \lambda\right)=F_{a b}+e_{a}^{\mu} e_{b}^{\nu} \bar{\psi}_{[\mu} \gamma_{\nu]} \lambda \\
\mathcal{D}_{\mu} \lambda= & \left(\partial_{\mu}-\frac{3}{2} b_{\mu}+\frac{1}{4} w_{\mu}^{a b} \gamma_{a b}-\frac{3}{2} i \gamma_{*} \mathcal{A}_{\mu}\right) \lambda \\
& -\left(\frac{1}{4} \gamma^{a b} \hat{F}_{a b}+\frac{1}{2} i \gamma_{*} D\right) \psi_{\mu} .
\end{aligned}
$$

Here, $e_{a}^{\mu}$ is the vierbein, with frame indices $a, b$ and coordinate indices $\mu, v$. The fields $w_{\mu}^{a b}, b_{\mu}$, and $\mathcal{A}_{\mu}$ are the gauge fields corresponding to Lorentz transformations, dilatations, and $T_{R}$ symmetry of the conformal algebra respectively, while $\psi_{\mu}$ is the gravitino. The covariant derivatives of other fields can be found in Eq. (4.6) of [11].

\section{A.3 Multiplication laws}

Given a set of (complex) multiplets $\mathcal{C}^{i}$, one can construct a new multiplet $\tilde{\mathcal{C}}=f\left(\mathcal{C}^{i}\right)$ with components

$$
\begin{aligned}
\tilde{\mathcal{C}}= & f \\
\tilde{\mathcal{Z}}= & f_{i} \mathcal{Z}^{i}, \\
\tilde{\mathcal{H}}= & f_{i} \mathcal{H}^{i}-\frac{1}{2} f_{i j} \overline{\mathcal{Z}}^{i} P_{L} \mathcal{Z}^{j}, \\
\tilde{\mathcal{K}}= & f_{i} \mathcal{K}^{i}-\frac{1}{2} f_{i j} \overline{\mathcal{Z}}^{i} P_{R} \mathcal{Z}^{j}, \\
\tilde{\mathcal{B}} & =f_{i} \mathcal{B}_{\mu}^{i}+\frac{1}{2} i f_{i j} \overline{\mathcal{Z}}^{i} P_{L} \gamma_{\mu} \mathcal{Z}^{j}, \\
\tilde{\Lambda}= & f_{i} \Lambda^{i}+\frac{1}{2} f_{i j}\left[i \gamma_{*} \mathcal{B}^{i}+P_{L} \mathcal{K}^{i}+P_{R} \mathcal{H}^{i}-\mathcal{D} \mathcal{C}^{i}\right] \mathcal{Z}^{j} \\
& -\frac{1}{4} f_{i j k} \mathcal{Z}^{i} \overline{\mathcal{Z}}^{j} \mathcal{Z}^{k}, \\
\tilde{\mathcal{D}}= & f_{i} \mathcal{D}^{i}+\frac{1}{2} f_{i j}\left(\mathcal{K}^{i} \mathcal{H}^{j}-\mathcal{B}^{i} \cdot \mathcal{B}^{j}-\mathcal{D} \mathcal{C}^{i} \cdot \mathcal{D} \mathcal{C}^{j}-2 \bar{\Lambda}^{i} \mathcal{Z}^{j}-\overline{\mathcal{Z}}^{i} \not \mathcal{D} \mathcal{Z}^{j}\right) \\
& -\frac{1}{4} f_{i j k} \overline{\mathcal{Z}}^{i}\left(i \gamma_{*} \mathcal{B}^{j}+P_{L} \mathcal{K}^{j}+P_{R} \mathcal{H}^{j}\right) \mathcal{Z}^{k} \\
& +\frac{1}{8} f_{i j k l} \overline{\mathcal{Z}}^{i} P_{L} \mathcal{Z}^{j} \overline{\mathcal{Z}}^{k} P_{R} \mathcal{Z}^{l} .
\end{aligned}
$$

For the multiplication laws involving real and (anti-)chiral multiplets, see section 5 of [11]. The bar on spinors are always the Majorana conjugate, $\bar{\chi}=\chi^{T} \hat{C}$, where $\hat{C}$ is the charge conjugation matrix satisfying $\hat{C} \gamma_{\mu} \hat{C}^{-1}=-\gamma_{\mu}^{T}$. We use this conjugate even if the spinor is not Majorana. This is the convention in [11].

\section{A.4 Components}

For convenience, we here summarise the multiplets in the seven-component notation. The multiplet $\mathcal{R}$ is made up of the following multiplets

$$
\begin{aligned}
& X=\left(\phi,-i \sqrt{2} P_{L} \chi,-2 F, 0,+i \mathcal{D}_{\mu} \phi, 0,0\right), \\
& S_{0}=\left(s_{0},-i \sqrt{2} P_{L} \Omega_{0},-2 F_{0}, 0,+i \mathcal{D}_{\mu} s_{0}, 0,0\right)
\end{aligned}
$$

$\bar{\lambda} P_{L} \lambda=\left(\bar{\lambda} P_{L} \lambda,-i \sqrt{2} P_{L} \Lambda, 2 D_{-}^{2}, 0,+i \mathcal{D}_{\mu}\left(\bar{\lambda} P_{L} \lambda\right), 0,0\right)$

$$
(V)_{D}=\left(D, \mathbb{D} \lambda, 0,0, \mathcal{D}^{b} \hat{F}_{a b},-\mathcal{D} \mathcal{D} \lambda,-\square^{C} D\right),
$$

and their charge conjugates. The chiral multiplet $w^{\prime 2}$ is defined by Eq. (3).

\section{B Fermion couplings}

This appendix presents the fermionic terms in the new FI contribution (21) up to the quadratic order in fermions. Concern- 
ing the fermion couplings, the contribution to the Lagrangian from (21) is given by [1]

$$
\begin{aligned}
\mathcal{L}_{\mathrm{FI}, \text { new }} / e= & -\frac{\xi}{2}\left[(\mathcal{R})_{\mathcal{D}} D-\mathcal{D}^{b} \hat{F}_{a b}(\mathcal{R})_{\mathcal{B}}^{a}-(\overline{\mathcal{R}})_{\lambda} \not D \lambda\right. \\
& \left.-\frac{1}{2} i D \psi \cdot \gamma \gamma_{*}(\mathcal{R})_{\lambda}+\cdots,\right],
\end{aligned}
$$

which is obtained by applying Eq. (11) to the definition $\mathcal{L}_{\mathrm{FI}, \text { new }} / e=-\xi\left[\mathcal{R} \cdot(V)_{D}\right]_{D}$ with

$\mathcal{R}=\left(S_{0} \bar{S}_{0} e^{-K / 3}\right)^{-3} \frac{\left(\bar{\lambda} P_{L} \lambda\right)\left(\bar{\lambda} P_{R} \lambda\right)}{T\left(\bar{w}^{\prime 2}\right) \bar{T}\left(w^{\prime 2}\right)}$,

and $(V)_{D}$ given by Eq. (2). We therefore need the $\mathcal{B}_{\mu}, \lambda$, and $D$ components of $\mathcal{R}$. Since we are interested in terms with two fermions in the new FI contribution, we need terms with up to two fermions for $(\mathcal{R})_{\mathcal{B}},(\mathcal{R})_{D}$, and terms with one fermion for $(\mathcal{R})_{\lambda}$. It therefore turns out that we need terms with up to two fermions for the lowest component of $T\left(\bar{w}^{\prime 2}\right)$, terms with one fermion for the fermionic (i.e. second) component of $T\left(\bar{w}^{\prime 2}\right)$, and terms with no fermions for the $F$-component of $T\left(\bar{w}^{\prime 2}\right)$. Let us denote these component fields by

$T\left(\bar{w}^{\prime 2}\right)=\left(C_{T}, P_{L} \chi_{T}, F_{T}\right)$.

Their explicit forms are given by

$$
\Delta^{2} C_{T}=-\left[\left(D_{+}^{2}\right)_{0 \mathrm{f}}+\overline{\mathbb{X}}_{2 \mathrm{f}}\right] \text {, }
$$

$$
\begin{aligned}
& \Delta^{2} P_{L} \chi_{T} \\
& \quad=P_{L}\left[-\mathbb{D}_{0 \mathrm{f}} \Lambda_{\mathrm{lf}}+2\left(\frac{\mathbb{D}_{0 \mathrm{f}} s_{0}}{s_{0}}-\frac{K_{\phi} \mathbb{D}_{0 \mathrm{f}} \phi}{3}\right) \Lambda_{\mathrm{lf}}-\frac{2 K_{\phi}\left(D_{+}^{2}\right)_{0 \mathrm{ff}}}{3} \chi\right], \\
& \Delta^{2} F_{T}=\left(\frac{2}{s_{0}} F_{0}-\frac{2 K_{\phi}}{3} F_{\phi}\right)\left(D_{+}^{2}\right)_{0 \mathrm{f}},
\end{aligned}
$$

where the subscript $2 \mathrm{f}$ indicates the two-fermion parts of the relevant term, and the same definition applies to the subscripts 0f, 1f. We also introduced the following symbols for compactness of the formulae,

$$
\begin{aligned}
& K_{\phi}=\frac{\partial K(\phi, \bar{\phi})}{\partial \phi}, \\
& \Delta=s_{0} \bar{s}_{0} e^{-K / 3}, \\
& P_{L} \Lambda=\sqrt{2} P_{L}\left(-\frac{1}{2} \gamma \cdot \hat{F}+i D\right) \lambda, \\
& D_{-}^{2}=D^{2}-\hat{F}^{-} \cdot \hat{F}^{-}-2 \bar{\lambda} P_{L} I D \lambda .
\end{aligned}
$$

Note that $\Delta=1$ under the Einstein frame gauge $s_{0}=\bar{s}_{0}=$ $e^{-K / 6}$. The symbol $\mathbb{X}_{2 \mathrm{f}}$ is minus the two-fermion part of the lowest component of $T\left(\bar{w}^{\prime 2}\right)$,

$$
\begin{aligned}
\mathbb{X}_{2 \mathrm{f}}= & -\left(\hat{F}^{-} \cdot \hat{F}^{-}\right)_{2 \mathrm{f}}-2 \bar{\lambda} P_{L}(\mathcal{D} \lambda)_{1 \mathrm{f}} \\
& +2\left(\frac{F_{0}}{s_{0}}-\frac{K_{\phi} F}{3}\right) \bar{\lambda} P_{L} \lambda+\frac{2}{3} K_{\phi} \bar{\chi} P_{L} \Lambda_{1 \mathrm{f}}
\end{aligned}
$$

Combining these results, we find that the components of $\mathcal{R}$ we need are given by

$$
\begin{aligned}
(\mathcal{R})_{B \mu} & =\Delta \frac{i}{\left(D_{-}^{2}\right)_{0 \mathrm{f}}\left(D_{+}^{2}\right)_{0 \mathrm{f}}} \bar{\Lambda}_{1 \mathrm{f}} P_{L} \gamma_{\mu} \Lambda_{1 \mathrm{f}} \\
(\mathcal{R})_{\lambda} & =\Delta \cdot \sqrt{2} i\left[\frac{P_{R} \Lambda_{1 \mathrm{f}}}{\left(D_{+}^{2}\right)_{0 \mathrm{f}}}-\frac{P_{L} \Lambda_{1 \mathrm{f}}}{\left(D_{-}^{2}\right)_{0 \mathrm{f}}}\right] \\
(\mathcal{R})_{D}= & \frac{2 \Delta}{\left(D_{-}^{2}\right)_{0 \mathrm{f}}}\left[\left(\frac{3 F_{0}}{s_{0}}-K_{\phi} F-\frac{\Delta^{2} F_{T}}{\left(D_{+}^{2}\right)_{0 \mathrm{f}}}\right) \bar{\lambda} P_{L} \lambda\right. \\
& \left.+\bar{\Lambda}_{1 \mathrm{f}} P_{L}\left(K_{\phi} \chi+\frac{\Delta^{2}}{\left(D_{+}^{2}\right)_{0 \mathrm{f}}} \chi_{T}\right)\right]+ \text { h.c. } \\
& +2 \Delta\left(\frac{\left(D_{-}^{2}\right)_{2 \mathrm{f}}-\mathbb{X}_{2 \mathrm{f}}}{\left(D_{-}^{2}\right)_{0 \mathrm{f}}}+\frac{\left(D_{+}^{2}\right)_{2 \mathrm{f}}-\overline{\mathbb{X}}_{2 \mathrm{f}}}{\left(D_{+}^{2}\right)_{0 \mathrm{f}}}-\frac{\bar{\Lambda}_{1 \mathrm{f}}(\mathcal{D} \Lambda)_{1 \mathrm{f}}}{2\left(D_{-}^{2}\right)_{0 \mathrm{f}}\left(D_{+}^{2}\right)_{0 \mathrm{f}}}\right)
\end{aligned}
$$

where we only kept two-fermion contributions in $(\mathcal{R})_{B \mu}$, $(\mathcal{R})_{D}$ and one-fermion contributions in $(\mathcal{R})_{\lambda}$. Note also that $\left(D_{-}^{2}\right)_{0 \mathrm{f}}=D^{2}-F^{-} \cdot F^{-}$.

\section{References}

1. D.Z. Freedman, A. Van Proeyen, Supergravity (Cambridge University Press, Cambridge, 2012)

2. P. Fayet, J. Iliopoulos, Spontaneously Broken Supergauge Symmetries and Goldstone Spinors. Phys. Lett. B 51, 461 (1974)

3. D.Z. Freedman, Supergravity with axial gauge invariance. Phys. Rev. D 15, 1173 (1977)

4. R. Barbieri, S. Ferrara, D.V. Nanopoulos, K.S. Stelle, Supergravity, R invariance and spontaneous supersymmetry breaking. Phys. Lett. B 113, 219 (1982)

5. R. Casalbuoni, S. De Curtis, D. Dominici, F. Feruglio, R. Gatto, Nonlinear realization of supersymmetry algebra from supersymmetric constraint. Phys. Lett. B 220, 569 (1989)

6. Z. Komargodski, N. Seiberg, From linear SUSY to constrained superfields. JHEP 0909, 066 (2009). arXiv:0907.2441 [hep-th]

7. I. Antoniadis, E. Dudas, S. Ferrara, A. Sagnotti, The VolkovAkulov-Starobinsky supergravity. Phys. Lett. B 733, 32 (2014). arXiv:1403.3269 [hep-th]

8. N. Cribiori, F. Farakos, M. Tournoy, A. Van Proeyen, FayetIliopoulos terms in supergravity without gauged R-symmetry. arXiv: 1712.08601 [hep-th]

9. I. Antoniadis, A. Chatrabhuti, H. Isono, R. Knoops, FayetIliopoulos terms in supergravity and D-term inflation. Eur. Phys. J. C 78(5), 366 (2018). arXiv:1803.03817 [hep-th]

10. S.M. Kuzenko, Taking a vector supermultiplet apart: alternative Fayet-Iliopoulos-type terms. arXiv:1801.04794 [hep-th] 
11. S. Ferrara, R. Kallosh, A. Van Proeyen, T. Wrase, Linear versus non-linear supersymmetry. JHEP 1604, 065 (2016). arXiv:1603.02653 [hep-th]

12. T. Kugo, $\mathrm{S}$. Uehara, $N=1$ Superconformal Tensor calculus: multiplets with external lorentz indices and spinor derivative operators. Prog. Theor. Phys. 73, 235 (1985)

13. Y. Aldabergenov, S.V. Ketov, Removing instability of inflation in Polonyi-Starobinsky supergravity by adding FI term. Mod. Phys. Lett. A 91(05), 1850032 (2018). arXiv:1711.06789 [hep-th]

14. F. Farakos, A. Kehagias, A. Riotto, Liberated $\mathcal{N}=1$ supergravity. JHEP 1806, 011 (2018). https://doi.org/10.1007/JHEP06. arXiv:1805.01877 [hep-th]
15. Y. Aldabergenov, S.V. Ketov, R. Knoops, General couplings of a vector multiplet in $\mathrm{N}=1$ supergravity with new FI terms. arXiv: 1806.04290 [hep-th]

16. S. Kachru, R. Kallosh, A.D. Linde, S.P. Trivedi, De Sitter vacua in string theory. Phys. Rev. D 68, 046005 (2003). arXiv:hep-th/0301240

17. I. Antoniadis, A. Kumar, T. Maillard, Magnetic fluxes and moduli stabilization. Nucl. Phys. B 767, 139 (2007). arXiv:hep-th/0610246

18. I. Antoniadis, J.-P. Derendinger, T. Maillard, Nonlinear N=2 Supersymmetry, effective actions and moduli stabilization. Nucl. Phys. B 808, 53 (2009). arXiv:0804.1738 [hep-th] 